\title{
Overview of two deterministic modelings for prompt emission in fission
}

\author{
Anabella Tudora \\ University of Bucharest, Faculty of Physics, 077125 Bucharest-Magurele, Romania
}

\begin{abstract}
Two models with a deterministic treatment of prompt emission in fission were developed at the University of Bucharest. Both models work with the same ranges of initial fragments and total kinetic energy and they use the same partition of the total excitation energy at full acceleration based on modelling at scission. The main difference between these modelings regards the prompt emission treatment itself. I.e. the Point-by-Point $(\mathrm{PbP})$ model uses a global treatment of sequential emission while the other modeling is based on an event-by-event treatment of sequential emission. Both models are submitted to a rigorous validation. This paper focuses on model results of different prompt $\gamma$ ray quantities, which describe very well the existing experimental data. A new method to calculate prompt $\gamma$-ray spectra, including a global treatment based on the distribution of prompt $\gamma$-ray energy per quanta, is proposed.
\end{abstract}

\section{Introduction}

Two deterministic modelings of prompt emission in fission were developed at the University of Bucharest. Chronologically the first developed model was Point-by-Point (PbP), results of this model being reported during the time in many publications, see e.g. Ref. [1] which includes a comprehensive description, as well as the references therein. During the time the $\mathrm{PbP}$ treatment has served to obtain the average values of input parameters for prompt emission models either based on the most probable fragmentation approach (the Los Alamos (LA) model) or on the multi-modal fission concept, i.e. a most probable fragmentation associated to each fission mode. The systematic of input parameters of the LA model [2] is based on the $\mathrm{PbP}$ treatment, too. The PbP model was also used for nuclear data evaluations (e.g. Ref. [3] and references therein), the investigation of even-odd effects in prompt emission (e.g. Ref. [4-6] and references therein) and for the prediction of $v(\mathrm{~A})$ at incident neutron energies (En) where multiple fission chances are involved [7, 8].

A second model of prompt emission was developed more recently [9], having as initial scope to obtain a general form of the residual temperature distribution $\mathrm{P}(\mathrm{T})$. The application of this model to 49 fission cases leaded to interesting systematic behaviours of different quantities characterizing the sequential prompt emission and the residual nuclei (resulting from the sequential emission) [10]. One of these systematics, i.e. the one regarding the ratios of the residual temperature (following the successive emission of each neutron) to the temperature of initial fragments (before prompt neutron emission) allowed to obtain a general form of the residual temperature distribution for each emission sequence and the inclusion of sequential emission into the LA model [10]. 
The main difference between these modelings regards the prompt emission itself. The $\mathrm{PbP}$ model uses a global treatment of sequential emission based on the residual temperature distribution on which the centre-of-mass energy spectrum of prompt neutrons at a given temperature is integrated. The other model $[9,11]$ includes a sequential emission treatment based on iterative equations of the residual temperature for each emission sequence associated to an initial fragment at a given value of TKE.

The similarities between these models consist of the use of the same range of initial fragmentations deterministically constructed and the same partition of the total excitation energy (TXE) based on modelling at scission. Other similarities and differences between these models, regarding the primary model results (i.e. multi-parametric matrices of different prompt emission quantities), the prescriptions used for the compound nucleus cross-sections of the inverse process of neutron evaporation from initial and residual fragments $\sigma_{\mathrm{c}}(\varepsilon)$ and the level density parameters of initial and residual fragments, as well as the input model parameters are mentioned in the next section.

The results of both models, which were reported up to now, mainly refer to prompt neutrons. For this reason this work focuses on model results of different prompt $\gamma$-ray quantities and their correlation with the prompt neutron multiplicity.

\section{Similarities and differences between these modelings}

Both models use the same fragmentation range deterministically constructed as follows [1, 9]. The mass range of initial fragments (before prompt neutron emission) is going from symmetric fission up to a very asymmetric split (with a step of 1 mass unit). For each mass number $A$ three or five charge numbers $Z$ are taken as the nearest integer values above and below the most probable charge $\mathrm{Zp}(\mathrm{A})$ taken as the unchanged charge distribution $\mathrm{Z}_{\mathrm{UCD}}(\mathrm{A})$ corrected with the charge polarization $\Delta \mathrm{Z}(\mathrm{A})$. For each fragment $\mathrm{A}, \mathrm{Z}$ the calculations are done at TKE values covering a large range (e.g. from 100 to $200 \mathrm{MeV}$ ) with a step size of 2 or $5 \mathrm{MeV}$.

Both models use the same TXE partition based on modelling at scission ([1, 3] and references therein). This consists of the calculation of the extra-deformation energy of initial fragments at scission with respect to the full acceleration and the partition of the available excitation at scission (obtained by subtracting the extra-deformation energies from TXE) between complementary nascent fragments under the assumption of statistical equilibrium at scission and fragment level density in the Fermi-gas regime.

The main difference in principle regards the treatment of sequential emission. The $\mathrm{PbP}$ model uses a global treatment of sequential emission which is based on the residual temperature distribution $\mathrm{P}(\mathrm{T})$. The prompt neutron spectrum in the centre-of-mass frame corresponding to an initial fragment $\mathrm{A}, \mathrm{Z}$ at a given TKE value is obtained by integrating the evaporation spectrum at a given temperature over $\mathrm{P}(\mathrm{T})$. The $\mathrm{PbP}$ computer code allows the use of different forms of $\mathrm{P}(\mathrm{T})$ either analytical expressions or numerical data (resulting from sequential emission calculations and provided as input files) [1].

The sequential emission modelling is based on recursive equations of residual temperature following the successive emission of each prompt neutron from each initial fragment $A$, $\mathrm{Z}$ of the fragmentation range at each value of the TKE range [9].

Different prescriptions for $\sigma_{\mathrm{c}}(\varepsilon)$ and the level density parameter can be used in both models. In the case of $\mathrm{PbP} \sigma_{\mathrm{c}}(\varepsilon)$ are provided by optical model calculations using phenomenological optical potential parameterisations adequate for nuclei appearing as fission fragments. The use of analytical expressions or even a constant $\sigma_{\mathrm{c}}$ is possible, too. In the case of sequential emission treatment an analytical expression of $\sigma_{\mathrm{c}}(\varepsilon)$ of initial and residual fragments 
(which depends on the s-wave neutron strength function provided by systematics) is currently employed (see Ref. [9] for details). The use of constant sc is also possible.

Usually energy-dependent level density parameters (provided by the super-fluid model) are used in the PbP model. In the case of sequential emission treatment, the transcendent successive equations of residual temperature can be solved only for non-energy dependent level density parameter prescriptions. In this treatment level density parameters provided by the Egidy-Bucurescu systematic for the BSFG model are used because they are close to the ones given by the super-fluid model for the majority of initial and residual fragments [9].

The primary results of the $\mathrm{PbP}$ model are multi-parametric matrices of different prompt emission quantities, generically labelled q(A,Z,TKE) (e.g. $v(\mathrm{~A}, \mathrm{Z}, \mathrm{TKE}),<\varepsilon>(\mathrm{A}, \mathrm{Z}, \mathrm{TKE})$, $\Phi(\varepsilon, \mathrm{A}, \mathrm{Z}, \mathrm{TKE}), \mathrm{E} \gamma(\mathrm{A}, \mathrm{Z}, \mathrm{TKE})$ etc.). The primary results of the sequential emission modelling are multi-parametric matrices of different quantities corresponding to each emission sequence $\mathrm{k}$ associated to an initial fragment $\mathrm{A}, \mathrm{Z}$ at a given TKE value, i.e. $\mathrm{q}_{k}$ (A,Z,TKE). Multiparametric matrices similar to the ones provided by the $\mathrm{PbP}$ model are obtained by averaging $\mathrm{q}_{k}(\mathrm{~A}, \mathrm{Z}, \mathrm{TKE})$ over the number of sequences corresponding to an initial fragment $\mathrm{A}, \mathrm{Z}$ at a given TKE value.

The secondary results of both modellings consist of single distributions of different prompt emission quantities $(\mathrm{q}(\mathrm{A}), \mathrm{q}(\mathrm{Z}), \mathrm{q}(\mathrm{TKE})$ ) and total average quantities $<\mathrm{q}>$. They are obtained by averaging the primary results $\mathrm{q}(\mathrm{A}, \mathrm{Z}, \mathrm{TKE})$ over fission fragment distributions $\mathrm{Y}(\mathrm{A}, \mathrm{Z}, \mathrm{TKE})$. These distributions are taken as $\mathrm{Y}(\mathrm{A}, \mathrm{Z}, \mathrm{TKE})=\mathrm{p}(\mathrm{Z}, \mathrm{A}) \mathrm{Y}(\mathrm{A}, \mathrm{TKE})$ in which the isobaric charge distribution $\mathrm{p}(\mathrm{Z}, \mathrm{A})$ is a Gaussian function centred on the most probable charge $\mathrm{Zp}(\mathrm{A})$ and $\mathrm{Y}(\mathrm{A}, \mathrm{TKE})$ are experimental data.

Regarding the input parameters: to provide the primary results (i.e. the multi-parametric matrices $\mathrm{q}(\mathrm{A}, \mathrm{Z}, \mathrm{TKE})$ ) both modellings do not use free or adjustable parameters. They need only data from recommended nuclear data libraries of RIPL1-3 (e.g. mass excesses, shell corrections, $\beta 2$ deformations, optical model parameterisations). This fact assures the possibility of prediction. For secondary results (i.e. average quantities as a function of A, of TKE etc.), experimental data of Y(A,TKE) are needed as input.

\section{Model validation based on prompt $\gamma$-ray results}

As it was already mentioned in Ref. [12], the main validation of a prompt emission model consists of the comparison of its primary results (i.e. multi-parametric matrices) with existing experimental data. This is a validation of the model itself because the Y(A,TKE) distribution is not involved.

The comparison of secondary model results (i.e. single distributions of different prompt emission quantities and total average quantities) with experimental data which are available in a much larger amount than in the case of multi-parametric data, is considered as a secondary validation of the prompt emission model together with the Y(A,TKE) distribution used [12].

The primary and secondary validations of both models reported up to now, e.g. in Refs. $[1,9,11,12]$, were mainly based on recent experimental data referring to prompt neutrons (e.g. the recent $v(\mathrm{~A}, \mathrm{TKE})$ data measured by Göök et al. for ${ }^{252} \mathrm{Cf}(\mathrm{SF})$ [13] and ${ }^{235} \mathrm{U}(\mathrm{n}, \mathrm{f})$ [14], the centre-of-mass energy spectrum $\Phi(\varepsilon)$ for the light and heavy fragment groups of ${ }^{235} \mathrm{U}(\mathrm{n}, \mathrm{f})$ [14] etc.), which were very well described by both model results.

For this reason the present paper focuses on the validation of both models based this time on prompt $\gamma$-ray results (obviously obtained concomitantly with the prompt neutron results, in the same run), which are compared with previous and recent prompt $\gamma$-ray data.

An example of primary validation is illustrated in Fig. 1 where the experimental prompt $\gamma$-ray energy matrix $\mathrm{E} \gamma(\mathrm{A}, \mathrm{TKE})$ of ${ }^{235} \mathrm{U}\left(\mathrm{n}_{\mathrm{th}}, \mathrm{f}\right)$ [15] (black symbols), in the 2D representations as $\mathrm{E} \gamma$ as a function of $\mathrm{A}$ for a given TKE value (upper part) and as $\mathrm{E} \gamma$ as a function of TKE 

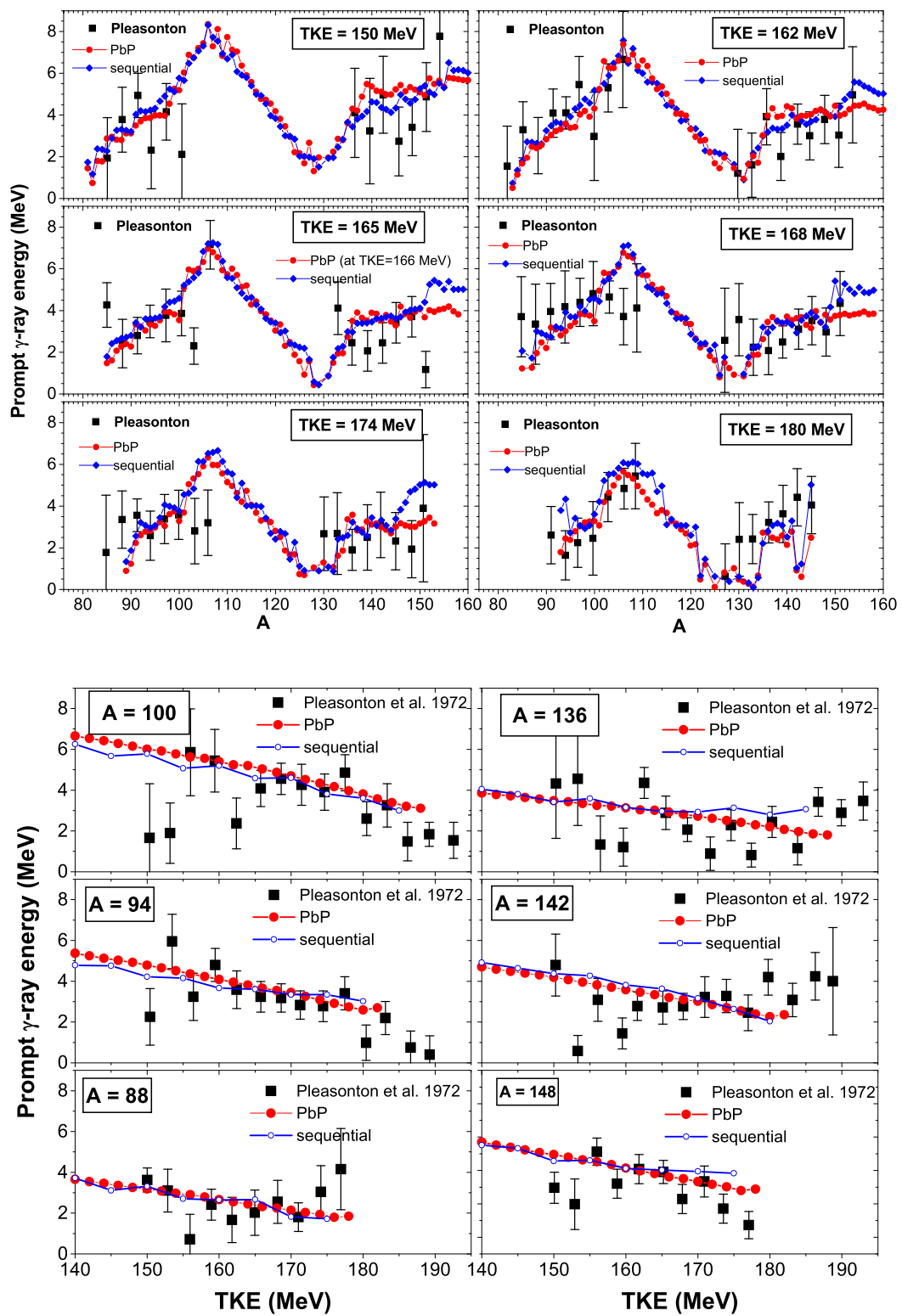

Figure 1. $\mathrm{E} \gamma(\mathrm{A}, \mathrm{TKE})$ results of $\mathrm{PbP}$ (red symbols) and sequential emission (blue symbols) calculations in comparison with the data of Pleasonton et al. [15] (black symbols) in the 2D representations as E $\gamma(\mathrm{A})$ at a given TKE value (upper part) and as $\mathrm{E} \gamma$ (TKE) for a given fragment mass (lower part).

for a given fragment mass (lower part), is well described by the $\mathrm{E} \gamma(\mathrm{A}, \mathrm{TKE})$ results of both models (red symbols for $\mathrm{PbP}$ and blue symbols for the sequential emission modelling).

Examples of secondary validation are given in Fig. 2, where the average prompt $\gamma$-ray energy and multiplicity as a function of A (left part) and as a function of TKE (right part) for ${ }^{235} \mathrm{U}\left(\mathrm{n}_{\mathrm{th}}, \mathrm{f}\right)$ provided by the PbP (red symbols) and sequential emission (blue symbols) calculations are in agreement with the experimental data (black and grey symbols). Another 


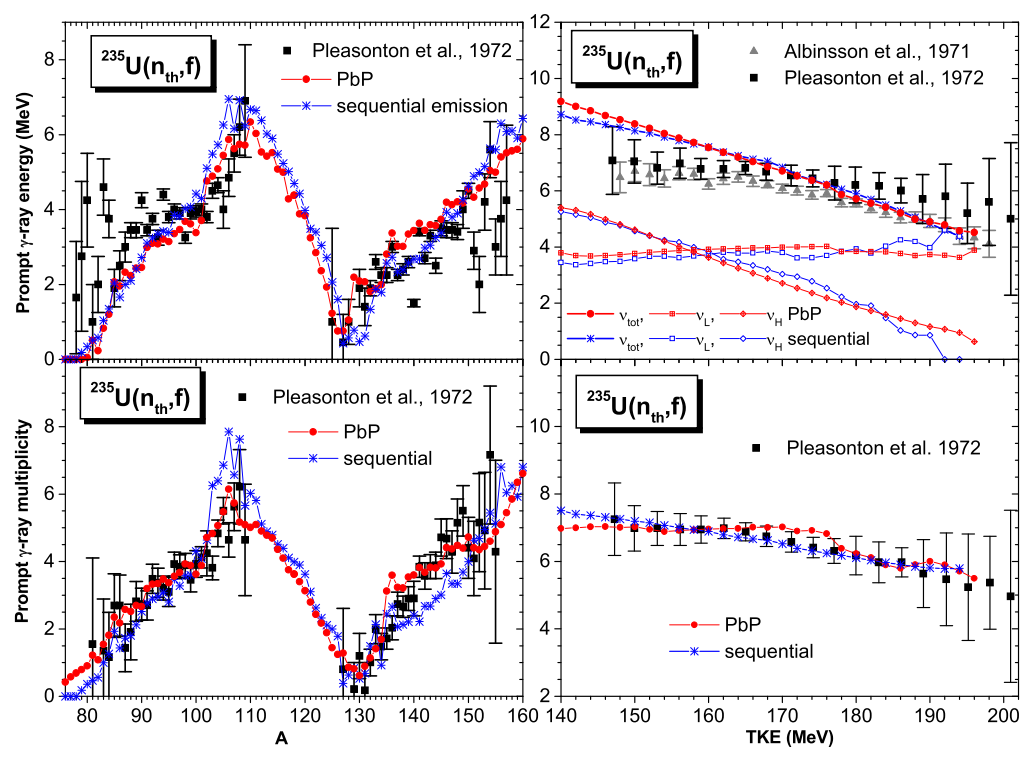

Figure 2. $\mathrm{E} \gamma(\mathrm{A}), \mathrm{N} \gamma(\mathrm{A}), \mathrm{E} \gamma(\mathrm{TKE})$ and $\mathrm{N} \gamma(\mathrm{TKE})$ results of $\mathrm{PbP}$ (red symbols) and sequential emission (blue symbols) for ${ }^{235} \mathrm{U}\left(\mathrm{n}_{\mathrm{th}}, \mathrm{f}\right)$ in comparison with experimental data (black and grey symbols).

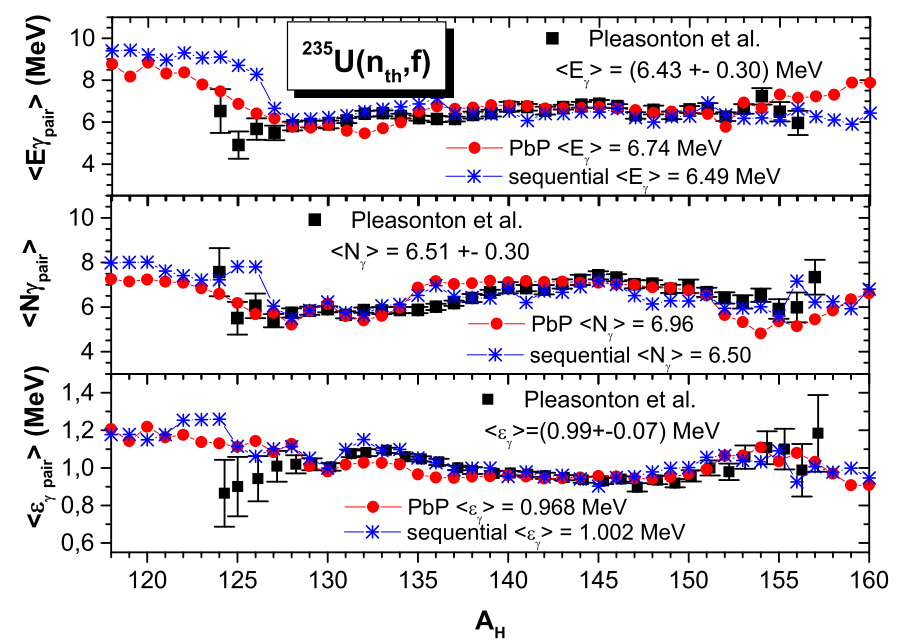

Figure 3. Experimental data of prompt $\gamma$-ray energy, multiplicity and energy per quanta of fragment pair for ${ }^{235} \mathrm{U}\left(\mathrm{n}_{\mathrm{th}}, \mathrm{f}\right)[15]$ (black symbols) and both model results (red and blue symbols, respectively).

example is given in Fig. 3 where the experimental data of the average prompt $\gamma$-ray energy, multiplicity and prompt $\gamma$-ray energy per quanta of the mass pair [15] are well described by the both model results, $\mathrm{PbP}$ and sequential emission.

Correlations between different prompt $\gamma$-ray quantities and the prompt neutron multiplicity were also investigated. The most known is the linear correlation between the prompt $\gamma$-ray energy and the prompt neutron multiplicity (which was reported during the time by many authors including us). For a nucleus fissioning spontaneously or at a given excitation energy, 

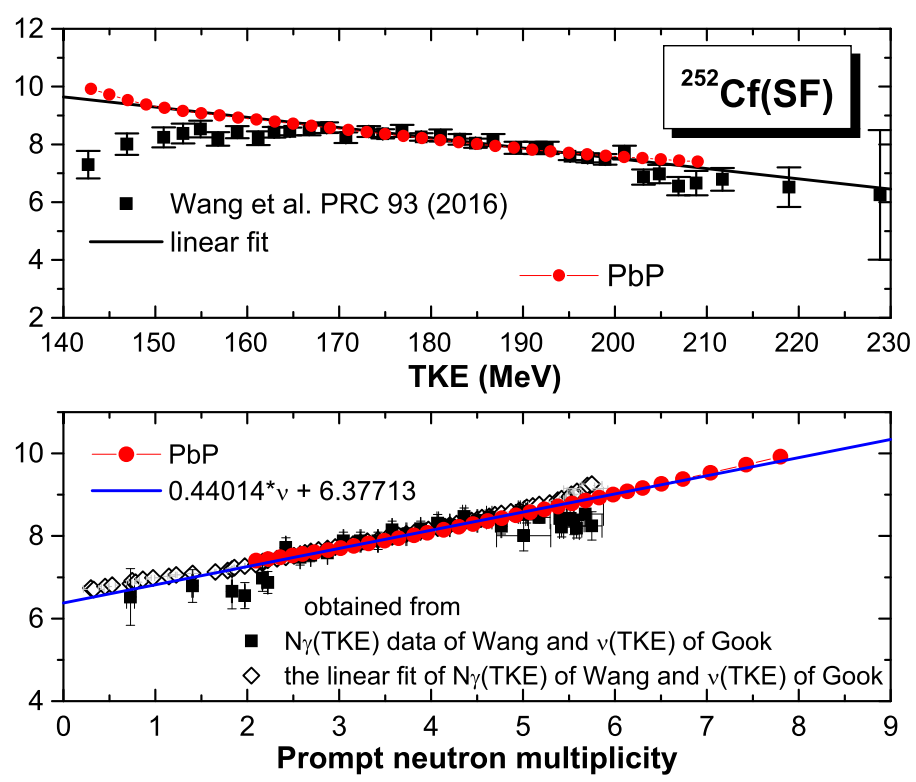

Figure 4. $\langle\mathrm{N} \gamma\rangle$ (TKE) (upper part) and the correlation $\langle\mathrm{N} \gamma\rangle(v)$ (lower part) for ${ }^{252} \mathrm{Cf}(\mathrm{SF})$ : the experimental data (full and open black symbols) and the $\mathrm{PbP}$ results (red circles).

this correlation is an obvious consequence of the linear decreasing behaviours of the average prompt neutron multiplicity and prompt $\gamma$-ray energy as a function of TKE.

The almost linear decrease of the average prompt $\gamma$-ray multiplicity as a function of TKE (exemplified for ${ }^{235} \mathrm{U}\left(\mathrm{n}_{\mathrm{th}}, \mathrm{f}\right)$ in the right lower part of Fig. 2 and for ${ }^{252} \mathrm{Cf}(\mathrm{SF})$ in the upper part of Fig. 4) together with the well known linear decrease of the average prompt neutron multiplicity as a function of TKE $[1,3,9,11-14]$ leads to a linear correlation between the prompt $\gamma$-ray multiplicity and the prompt neutron multiplicity, as it can be seen in the lower part of Fig. 4 for ${ }^{252} \mathrm{Cf}(\mathrm{SF})$ taken as an example.

The experimental data plotted in the lower part of Fig. 4 (with full and open black symbols) were obtained from the experimental $\mathrm{N} \gamma(\mathrm{TKE})$ data of Wang et al. [16] and their linear fit respectively, and the experimental $\langle v\rangle$ (TKE) data of Göök et al. [13]. As it can be seen, these data exhibit an almost linear behaviour well described by the PbP result (red circles).

Another correlation, illustrated in Fig. 5 for the case of ${ }^{252} \mathrm{Cf}(\mathrm{SF})$, regards the linear behaviour of $\sigma_{\gamma}^{2}=<\mathrm{E} \gamma^{2}>-<\mathrm{E} \gamma^{2}>$ as a function of prompt neutron multiplicity.

The experimental data plotted with black squares in Fig. 5 were obtained from the experimental data $\mathrm{N} \gamma$ (TKE) of Wang et al. [16], E $\gamma$ (TKE) of Nifenecker et al. [17] and $v$ (TKE) of Göök et al. [13] using the relation $\left.\sigma_{\gamma}{ }^{2}=2<\mathrm{E} \gamma\right\rangle^{2} / \mathrm{N}<\gamma>$ (based on the assumption of a Poisson distribution of $\mathrm{N} \gamma$ ). As it can be seen these data are well described by the $\mathrm{PbP}$ result (red circles) which exhibits an obvious linear behaviour.

As an alternative to the statistical Hauser-Feshbach calculation of prompt $\gamma$-ray emission in competition with the neutron emission from many nuclei appearing as fission fragments (a great part of these nuclei having scarce or unknown level schemes) requiring a long computing time, a more simple method to calculate the prompt $\gamma$-ray spectrum (adequate for evaluation purposes) is proposed. This method consists of a global treatment following an idea which is similar to the one based on $\mathrm{P}(\mathrm{T})$ which is used for the prompt neutron spectrum. This method is based on the distribution of prompt $\gamma$-ray energy per quanta $\mathrm{D}(\varepsilon \gamma)$ of the light 


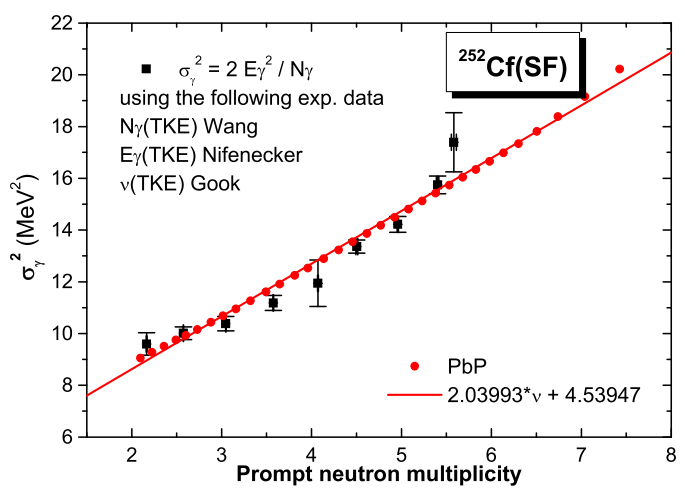

Figure 5. $\sigma_{\gamma}{ }^{2}$ as a function of prompt neutron multiplicity for the case of ${ }^{252} \mathrm{Cf}(\mathrm{SF})$.

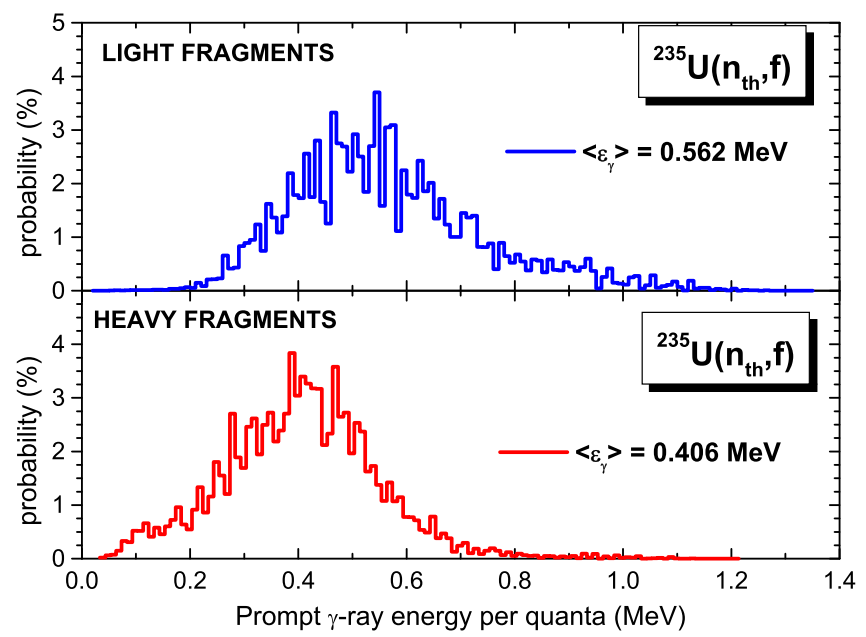

Figure 6. Distributions of prompt $\gamma$-ray energy per quanta corresponding to the light and heavy fragment groups of ${ }^{235} \mathrm{U}\left(\mathrm{n}_{\mathrm{th}}, \mathrm{f}\right)$ resulting from the $\mathrm{PbP}$ treatment.

and heavy fragments on which the prompt $\gamma$-ray spectrum at a given prompt $\gamma$-ray energy per quanta $\varphi(\varepsilon \gamma, \mathrm{E} \gamma)$ is integrated, the result being multiplied with the average prompt $\gamma$-ray multiplicity of the light and heavy fragment groups, i.e.:

$$
\Phi\left(E_{\gamma}\right)=\bar{N}_{\gamma L} \int_{0}^{\varepsilon \gamma_{\max L}} D_{L}\left(\varepsilon_{\gamma}\right) \varphi\left(\varepsilon_{\gamma}, E_{\gamma}\right) d \varepsilon_{\gamma}+\bar{N}_{\gamma H} \int_{0}^{\varepsilon \gamma_{\max H}} D_{H}\left(\varepsilon_{\gamma}\right) \varphi\left(\varepsilon_{\gamma}, E_{\gamma}\right) d \varepsilon_{\gamma}
$$

Distributions of prompt $\gamma$-ray energy per quanta entering Eq. (1) are exemplified in Fig. 6 for the case of ${ }^{235} \mathrm{U}\left(\mathrm{n}_{\mathrm{th}}, \mathrm{f}\right)$. They were obtained from the primary PbP result of $\varepsilon \gamma(\mathrm{A}, \mathrm{Z}, \mathrm{TKE})$ and the fragment distribution of Ref. [18].

Figure 7 shows preliminary results of prompt $\gamma$-ray spectrum based on Eq. (1) (red lines) exemplified for ${ }^{235} \mathrm{U}\left(\mathrm{n}_{\mathrm{th}}, \mathrm{f}\right)$ (upper part) and ${ }^{239} \mathrm{Pu}\left(\mathrm{n}_{\mathrm{th}}, \mathrm{f}\right)$ (lower part). As it can be seen they give an overall good description of the experimental data for ${ }^{235} U\left(n_{t h}, f\right)$ measured by Oberstedt et al. [19] (i.e. five data sets taken from EXFOR [20], with the entries specified in 


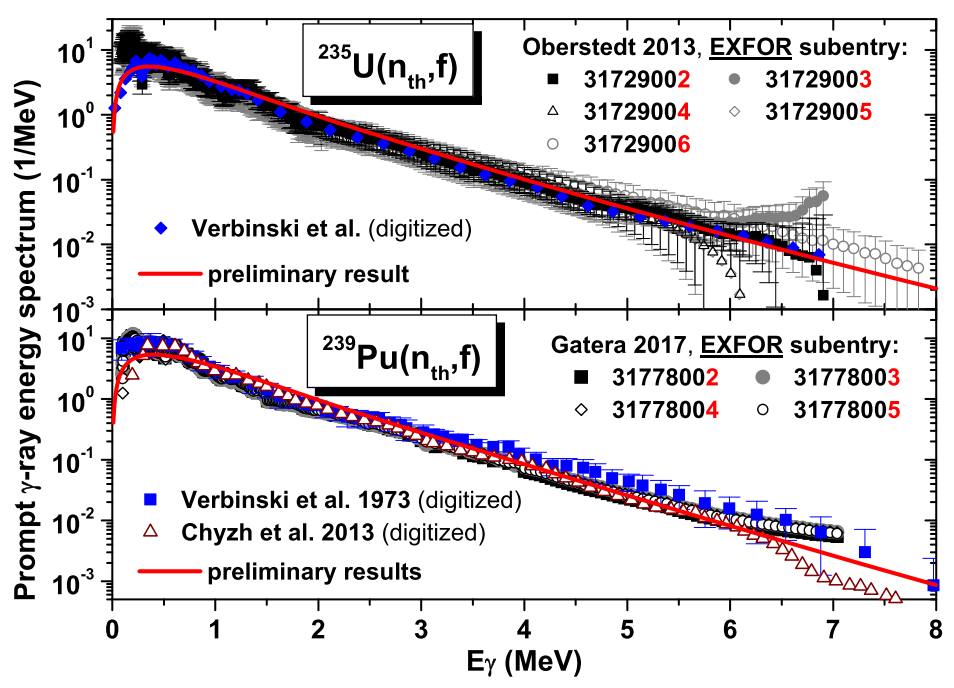

Figure 7. Preliminary prompt $\gamma$-ray spectrum results (red lines) for ${ }^{235} \mathrm{U}\left(\mathrm{n}_{\mathrm{th}}, \mathrm{f}\right)$ (upper part) and ${ }^{239} \mathrm{Pu}\left(\mathrm{n}_{\mathrm{th}}, \mathrm{f}\right)$ (lower part) in comparison with the experimental data [19-24] (different full and open symbols).

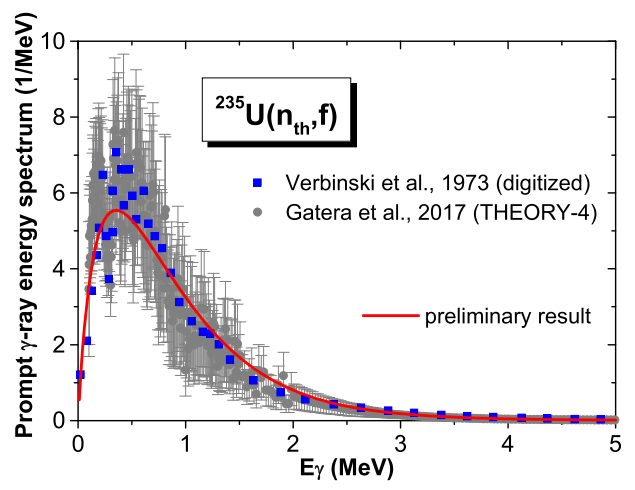

Figure 8. Focus of the low energy part of the prompt $\gamma$-ray spectrum of ${ }^{235} \mathrm{U}\left(\mathrm{n}_{\mathrm{th}}, \mathrm{f}\right)$.

the figure legend, which correspond to the measurements using five different detectors) and Verbinski et al. [21] and for ${ }^{239} \mathrm{Pu}\left(\mathrm{n}_{\mathrm{th}}, \mathrm{f}\right)$ measured by Gatera et al. [22] taken from EXFOR [20] (also with the entries of data sets given in the figure legend), Verbinsky et al. [21] and Chyzh et al. [23].

A focus of the low energy part of the prompt $\gamma$-ray spectrum is illustrated in Fig. 8 where the calculated spectrum of ${ }^{235} \mathrm{U}\left(\mathrm{n}_{\mathrm{th}}, \mathrm{f}\right)$ is compared with recent experimental data [24] (grey symbols). A slight underestimation of a part of experimental data, but remaining inside the error bars, is observed at low prompt $\gamma$-ray energies below $1 \mathrm{MeV}$. 


\section{Conclusions}

Both deterministic models of prompt emission ( $\mathrm{PbP}$ with a global treatment of sequential emission and a model with a detailed treatment of sequential emission) were submitted to a rigorous and detailed validation including:

i) The validation of the model itself: i.e. a very good description of multi-parametric experimental data (e.g. recent $v(\mathrm{~A}, \mathrm{TKE})$ data and the existing data of $\mathrm{E} \gamma(\mathrm{A}, \mathrm{TKE})$ ).

ii) The validation of the model together with a fragment distribution Y(A,TKE): i.e. a very good description of experimental single distributions and total average quantities related to prompt neutrons (e.g. $v(\mathrm{~A}), v(\mathrm{TKE}),<\varepsilon>(\mathrm{A}),<\varepsilon>(\mathrm{TKE}), \mathrm{P}(v), \Phi(\varepsilon)$ etc.) and to prompt $\gamma$-rays (e.g. E $\gamma(\mathrm{A}), \mathrm{E} \gamma(\mathrm{TKE}), \mathrm{N} \gamma(\mathrm{A}), \mathrm{N} \gamma(\mathrm{TKE}),\langle\mathrm{E} \gamma\rangle,\langle\mathrm{N} \gamma\rangle,\langle\varepsilon \gamma\rangle, \Phi(\mathrm{E} \gamma)$ etc.)

Correlations between the prompt neutron multiplicity and different prompt $\gamma$-ray quantities were emphasized, too.

The preliminary results of prompt $\gamma$-ray spectrum provided by a simple modelling including a global treatment based on the distribution of prompt $\gamma$-ray energy per quanta (provided by the $\mathrm{PbP}$ model) give an overall good description of the prompt $\gamma$-ray spectrum data measured for ${ }^{235} \mathrm{U}\left(\mathrm{n}_{\mathrm{th}}, \mathrm{f}\right),{ }^{239} \mathrm{Pu}\left(\mathrm{n}_{\mathrm{th}}, \mathrm{f}\right)$ and ${ }^{252} \mathrm{Cf}(\mathrm{SF})$.

The determination of a general analytical form for the distribution of prompt $\gamma$-ray energy per quanta (as in the case of the triangular residual temperature distribution $\mathrm{P}(\mathrm{T})$ used in the prompt neutron spectrum calculation) is in progress.

This work was done in the frame of the Romanian Project PN-III-P4_PCE-2016-0014 (contract No. 7/2017).

\section{References}

[1] A. Tudora, F.-J. Hambsch, Eur. Phys. J. A 53, 159 (2017)

[2] A. Tudora, Ann. Nucl. Energy 36, 72-84 (2009)

[3] R. Capote et al., Nucl. Data Sheets 131, 1-106 (2016)

[4] A. Tudora, F.-J. Hambsch, G. Giubega, Eur. Phys. J. A 52, 182 (2016)

[5] A.Tudora, F.-J. Hambsch, G. Giubega, I. Visan, Rom. Rep. Phys. 68, 571-581 (2016)

[6] G. Giubega, I. Visan, A. Tudora, Rom. Rep. Phys. 68 1024-1034 (2016)

[7] A. Tudora, F.-J. Hambsch, V. Tobosaru, Phys. Rev. C 94, 044601 (2016)

[8] A. Tudora, F.-J. Hambsch, V. Tobosaru, Nuc. Sci. Eng. 192, 52-69 (2018)

[9] A. Tudora, F.-J. Hambsch, V. Tobosaru, Eur. Phys. J. A 54, 87 (2018)

[10] A. Tudora, "Sistemtic behaviours of different quantities related to sequential prompt emission in fission", ND-2019 Int. Conf. on Nuclear Data for Science and technology, May 2019, Beijing, China, proceeding in press

[11] A. Tudora, A. Matei, Rom. J. Phys. 64 (1-2) 301 (2019)

[12] A. Tudora, Eur. Phys. J. A 55, 98 (2019)

[13] A. Göök, F.-J. Hambsch, M. Vidali, Phys. Rev. C 90, 064611 (2014)

[14] A. Göök, F.-J. Hambsch, S. Oberstedt, M. Vidali, Phys. Rev. C 98, 044615 (2018)

[15] F. Pleasonton, R.L. Ferguson, H.W. Schmitt, Phys. Rev. C 6, 1023-1039 (1972)

[16] T. Wang et al., Phys. Rev. C 93, 014606 (2016)

[17] H. Nifenecker, C. Signarbieux, R. Rabinet, J. Poitou, "Neutron and gamma emission in fission" (review paper), IAEA-SM-174/207, 117-178 (1973)

[18] A. Al-Adili, F.-J. Hambsch, S. Pomp, S. Oberstedt, Phys. Rev. C 86, 054601 (2012)

[19] A. Oberstedt et al., Phys. Rev. C 87, 051602 (2013) 
[20] EXFOR Experimental Nuclear Data Library (available online at https://wwwnds.iaea.org) nucleus U-235, reaction (n,f), entries 31729002-31729006 (Oberstedt et al.); nucleus $\mathrm{Pu}-239$, reaction (n,f), entries 31778002-31778005 (Oberstedt et al.)

[21] V. V. Verbinski, H. Weber, R. E. Sund, Phys. Rev. C 7, 1173-1185 (1973)

[22] A. Gatera et al., Phys. Rev. C 95, 064609 (2017)

[23] A. Chyzh et al., Phys. Rev. C 87, 034620 (2013)

[24] A. Gatera et al., EPJ Web of Conferences 169, 00003 (2018) 